\title{
Cytology and Quantitative Analysis of Aposporous Embryo Sac Development in Guineagrass (Panicum maximum Jacq.)
}

\author{
LanZhuang Chen and Teruo Kozono \\ Laboratory of Plant Genetic Resources, Kyushu National Agricultural Experiment Station, \\ Miyazaki, 880, Japan
}

Accepted May 11, 1994

Apomixis is a reproduction mode that bypasses female meiosis and syngamy to produce embryos genetically identical to the maternal parent (Asker and Jerling 1992). Apomixis not only represents an interesting reproduction mode that can result in the production of 4- and 8nucleate embryo sacs simultaneously, i.e. in Panicum maximum (guineagrass), but also provides a method for cloning plants through seeds (Hanna and Bashaw 1987).

Three major mechanisms of apomixis, adventitious embryony, apospory and diplospory, have been identified based upon the origin and development of cells from which the embryo derives (Hanna and Bashaw 1987). In guineagrass aposporous apomixis with pseudogamy is the mechanism of apomictic reproduction (Warmke 1954). Furthermore, research on the reproduction mode in guineagrass has been conducted to reveal the mode of inheritance of apomixis in sexual (Smith 1972, Hanna et al. 1973) and in apomixis accessions (Warmke 1954, Savidan 1975, Savidan and Pernes 1981, Nakajima and Mochizuki 1983, Nakagawa 1990). Ozias-Akins et al. (1993) have demonstrated co-inheritance of apomictic reproduction and two molecular markers in Pennisetum. These studies have indicated apomixis being mainly controlled by a single dominant or a few tightly linked genes.

In guineagrass, although some research has been reported on cytology and inheritance of apomixis, the period of apomictic gene expression, the key to cloning the apomixis genes has not been identified. The appearance of aposporous embryo sac initial cells (AESIC), from which aposporous embryo sac is derived, should be considered the most relevant stage for the expression of apomixis genes, because these cells appear only in apomictic but not in sexual plants. However, the mechanism of AESIC development is not well understood. If an index is found through cytological evidence, it can be used to estimate the range of period of AESIC appearance. The present study focuses on cytological observations of AESIC appearance and development, and using ovary length as an index, estimates the period of AESIC appearance to get information on the best point of time for sampling materials for apomixis gene isolation program.

\section{Materials and methods}

Seven facultatively apomictic accessions and three obligately sexual accessions collected from Tanzania, Kenya and Japan, were used in this study (Table 1). Since Tanzania accessions could not bloom under field condition in Miyazaki, the accessions were all transplanted into a greenhouse. One hundred to 300 buds or flowers before and at anthesis were collected per accession for embryo sac analysis.

Embryo sacs were observed by the method of Kojima and Nagato (1992). The buds and flowers collected were fixed in $\mathrm{FPA}_{50}$ (formalin : propionic acid : $50 \%$ ethanol $=5: 5: 90$ ) for 5- 
Table 1. Number of embryo sacs per ovule and the frequency of apospory in Panicum maximum

\begin{tabular}{|c|c|c|c|c|c|}
\hline \multirow{2}{*}{ Accession } & \multirow{2}{*}{$2 n$} & \multirow{2}{*}{$\begin{array}{c}\text { No. ovules } \\
\text { observed }\end{array}$} & \multirow{2}{*}{$\begin{array}{l}\text { Mean no. embryo } \\
\text { sacs (Range) }\end{array}$} & \multicolumn{2}{|c|}{ Frequency of apospory $\%$} \\
\hline & & & & Ist e.s. & 2nd e.s. ${ }^{4)}$ \\
\hline \multicolumn{6}{|l|}{ Apomictic } \\
\hline Green panic & 32 & 60 & $2.5(1-5)$ & 95 & 94 \\
\hline N68 $96-8-0-11^{1)}$ & 32 & 57 & $2.5(1-6)$ & 94 & 81 \\
\hline Natsuyutaka & 32 & 60 & $2.5(1-6)$ & 90 & 90 \\
\hline$T-41^{2)}$ & 32 & 52 & $2.4(1-4)$ & 84 & 81 \\
\hline $\mathrm{T}-75^{2)}$ & 32 & 54 & $2.2(1-5)$ & 72 & 65 \\
\hline Natsukaze & 32 & 72 & $1.6(1-4)$ & 68 & 72 \\
\hline$T-2^{2 l}$ & 32 & 63 & $1.6(1-4)$ & 59 & 67 \\
\hline \multicolumn{6}{|l|}{ Sexual } \\
\hline $\mathrm{T}-5^{21}$ & 16 & 58 & $1.0(1-1)$ & 0 & 0 \\
\hline$T-36^{2)}$ & 16 & 48 & $1.0(1-1)$ & 0 & 0 \\
\hline N68/96-8' & 32 & 60 & $1.0(1-1)$ & 0 & 0 \\
\hline
\end{tabular}

"The accessions were collected from Kenya; ${ }^{2}$ The accessions were from Tanzania; ${ }^{3}$ Embryo sacs in micropylar end; ${ }^{4}$ Embryo sacs in position nearest to the 1 st ones.

7 days at $4^{\circ} \mathrm{C}$. Then, ovaries were isolated from fixed buds or flowers, cleared in Herr's benzyl-benzate-four-and-a-half fluid (Herr 1982) for over $2 \mathrm{hr}$ at $0-4^{\circ} \mathrm{C}$, and observated with Nomarski differential interference-contrast optics. To estimate the period of AESIC appearance, the ovary length was measured and used as an index. The length of ovary, from the top where the style branches being separated, to the position adjacent to placenta, was measured with ocular micrometer. And then, a quantitative analysis for ovary length of various stages was carried out. The frequency of apospory was calculated at anthesis, based on the behavior of micropylar embryo sac (1st embryo sac) and the embryo sac (2nd embryo sac) nearest to the 1 st embryo sac, in other words, based on the ratio of apomictic embryo sac (4-nucleate): sexual embryo sac (8- or 5-nucleate).

\section{Results}

1. Megasporogenesis. In sexual and apomictic accessions similar behavior in megasporogenesis was observed. The megaspore mother cell underwent meiosis to form a linear tetrad, of which the three micropylar cells degenerated at an early stage, and only the chalazal one enlarged and formed a functional megaspore (Fig. 1A).

2. Embryo sac formation in sexual ovules. In sexual plants the functional megaspore continued to divide three times and formed a mature Polygonum-type embryo sac (8-nucleate and 7-celled): egg apparatus with one egg cell, two synergid cells in micropylar end; three antipodal cells in chalazal end, and a central cell with 2 polar nuclei (Fig. 1B, C).

3. Appearance of AESIC. Apomictic accessions, in contrast, showed embryo sac formation different from sexual ones. AESIC derived from enlarged unreduced nucellar cells appeared at first at the micropylar end while the functional megaspore was usually still existing at the chalazal end (Fig. $2 \mathrm{~A}_{1}, \mathrm{~A}_{2}$ ). The AESIC was distinguishable with its complete nuclear membrane, shorter and more vacuolated cell type. Megaspores often were without clear membranes.

4. AESIC-derived embryo sac formation. In general, the AESIC followed a special course to form embryo sac. It undergoes mitosis two times and forms 2-, 4-, and a mature Panicum-type embryo sac (4-nucleate and 4-celled), containing one egg cell, two synergid cells and one polar nucleus (Fig. 2B, C, D). In most ovules multiple apomictic embryo sacs were observed (Fig. 2E). The characteristics of mature apomictic embryo sacs are as following: 1) 


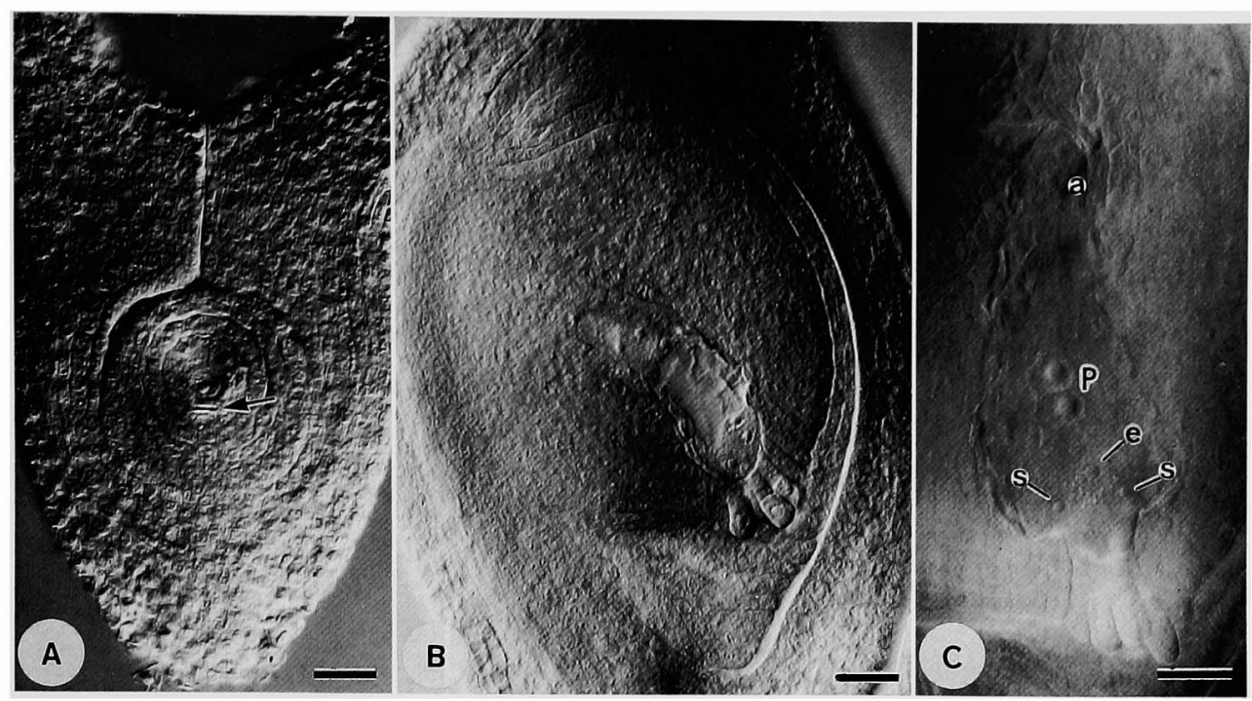

Fig. 1. Sexual embryo sac formation in facultatively apomictic guineagrass (Panicum maximum). (A) functional megaspore and degeneration of the other three megaspores (arrow), (B) eight-nucleate sexual embryo sac, (C) mature sexual embryo sac. $a=$ antipodals; $p=$ polar nuclei; $\mathrm{e}=$ egg cell; s=synergids; Fig. $1 \mathrm{~A}$ and Fig. 1B, bar $=50 \mu \mathrm{m}$; Fig. 1C, bar $=100 \mu \mathrm{m}$.

egg cell, its nucleolus, ca. $8 \mu \mathrm{m}$ in diameter, being visible and surrounded with bright starch grains, with which sometimes the nucleus and its membrane could not be distinguished, and cytoplasma was denser than the other cells; 2) synergid cells, its nucleolus, ca. $4 \mu \mathrm{m}$ in diameter, usually observed in either synergid, the cytoplasma was very few and the cell was occupied with bigger vacuoles; 3 ) polar nucleus, the nucleolus, ca. $12 \mu \mathrm{m}$ in diameter, surrounded by nuclear membrane, and cytoplasma was few; 4) no antipodals.

Appearance of filiform apparatus is also an evidence of embryo sac maturity in both sexual and apomictic plants. In most embryo sacs of apomictic ovules, each cell contains only one nucleus, and each nucleus contains one nucleolus during embryo sac formation. So were the sexual ovules but there were two nuclei in central cell. The nucleoli are stained deeply with the clearing fluid, so that we can easily distinguish the stage of embryo sac formation by counting the numbers of nucleoli. Apomictic embryo sac in micropylar end, matured about one day before anthesis, but in sexual accessions was the day at anthesis.

5. Estimation of the period of AESIC appearance. In most ovules of apomictic plants a number of AESICs appeared in same ovule. In this case, the 1st AESIC always located in micropylar end. The numbers of AESICs were increased as the ovary grew in length. For example, the ovary length in N68/96-8-0-11 was $384 \mu \mathrm{m}$ when the 1st AESIC appeared, and it became $544 \mu \mathrm{m}$ when the 5 th one did. So were the other accessions (Table 2). One-hundred and sixty $\mu \mathrm{m}, 162 \mu \mathrm{m}$ and $146 \mu \mathrm{m}$ were the difference in ovary length, from the first to the last AESIC, of N68/96-8-0-11, Green panic and T-2, respectively. These values also indicated that AESICs in the same ovules seemed not to differentiate synchronously.

Within the same facultatively apomictic accessions, the time of maturity of both sexual and aposporous embryo sacs located in micropylar end showed similar values of ovary length, although all of sexual embryo sacs were little shorter than aposporous ones (Table 2).

6. Degeneration of embryo sac. Some ovules had completely degenerated embryo sacs. The length range of ovaries containing degenerated embryo sac was between that of ovaries staged from 4-nucleate to their embryo sac maturity in apomictic plants, and from 8-nucleate to embryo sac maturity in sexual plants, respectively (Table 2). On the other hand, functional 


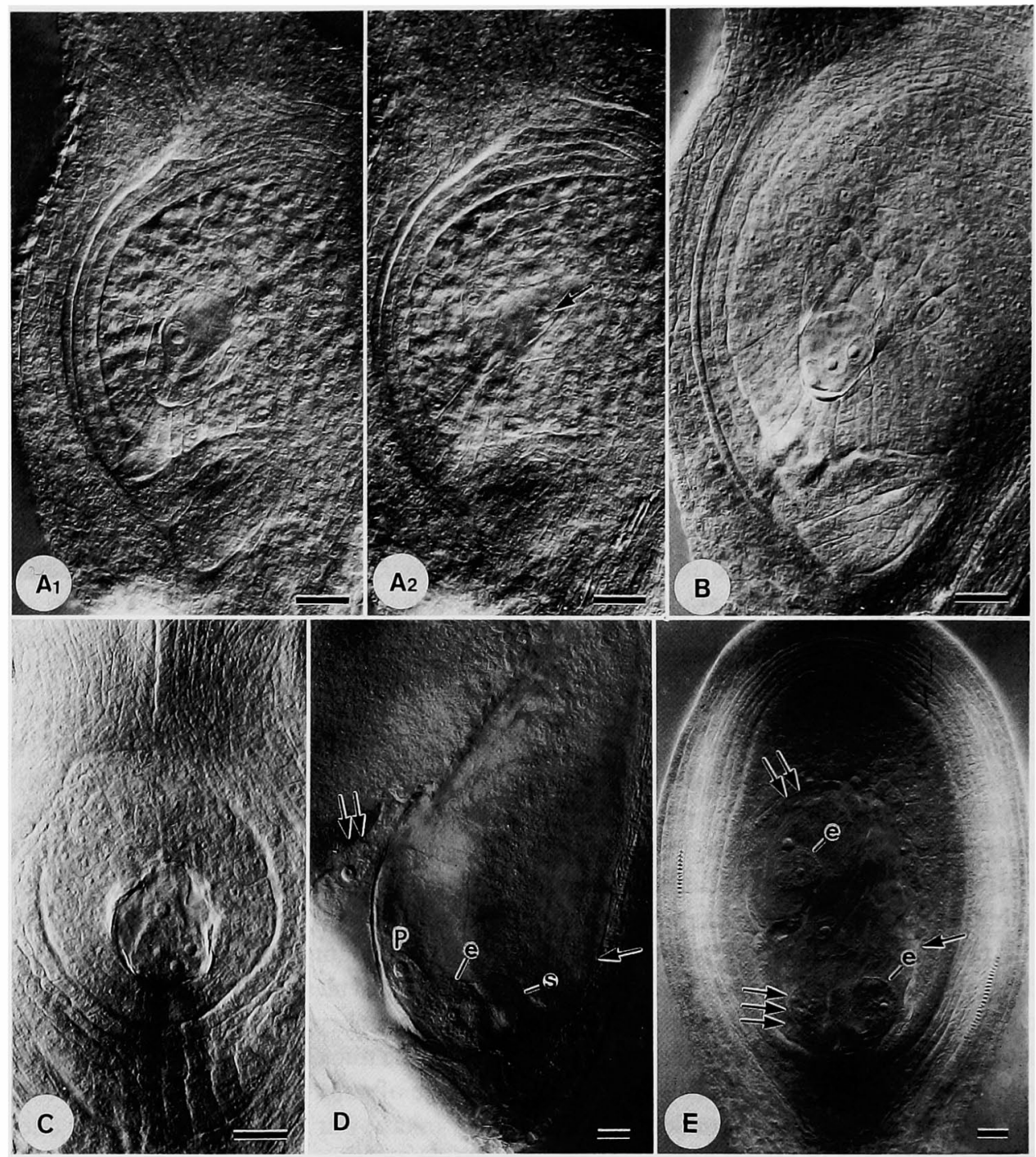

Fig. 2. Apomictic embryo sac formation in facultatively apomictic guineagrass (Panicum maximum $)$. $\left(\mathrm{A}_{1}\right)$ appearance of $\mathrm{AESIC}$ in micropylar end and $\left(\mathrm{A}_{2}\right)$ remaining of functional megaspore without nuclear membrance in chalazal end (arrow) in one ovule (Fig. $2 \mathrm{~A}_{1}$ and Fig. $2 \mathrm{~A}_{2}$, Two focal planes of the same specimen), (B) two-nucleate apomictic embryo sac, (C) four-nucleate apomictic embryo sac, (D) mature apomictic embryo sac in micropylar end (single arrow) and polar nucleus in another apomictic embryo sac (double arrows), (E) polyembryonic ovule: mature apomictic embryo sac in micropylar end (single arrow), another mature apomictic embryo sac (double arrows), and 4-nucleate-stage apomictic embryo sac (three arrows); $\mathrm{p}=$ polar nucleus; $\mathrm{e}=$ egg cell; $\mathbf{s}=$ synergid; bar $=50 \mu \mathrm{m}$.

megaspore stage showed a range of ovary length so wide that the ovary length became very close to the value of ovaries degenerated.

7. Relationship between the frequency of apospory and the number of embryo sacs. A wide range in frequency of apospory, from 59\% to $95 \%$ in first embryo sac, and from $65 \%$ to $94 \%$ in the second ones was obtained (Table 1). In the accessions with lower apospory frequency, such as Natsukaze and T-2, the frequencies of apospory in second embryo sac were higher than that in first embryo sac. From Table 1, it is seen that accessions with a higher 


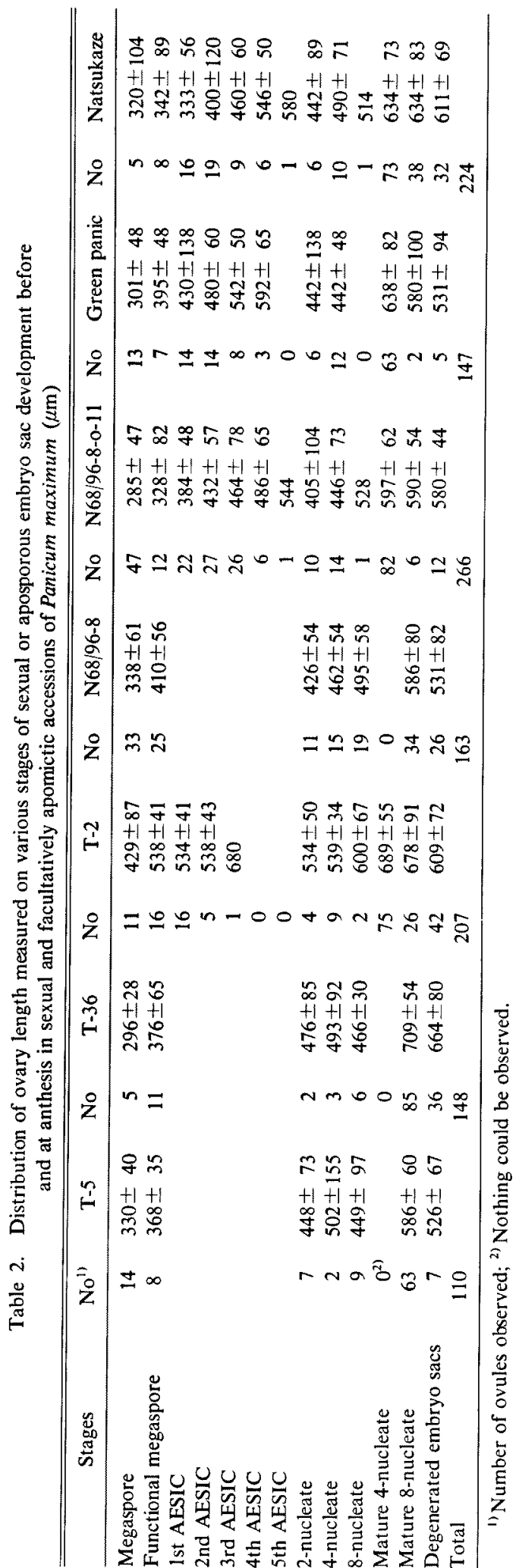


Table 3. Type and number of embryo sacs at anthesis in apomictic Panicum maximum

\begin{tabular}{lccccccc}
\hline \multirow{2}{*}{ Accession } & \multirow{2}{*}{$\begin{array}{c}\text { No ovules } \\
\text { observed }\end{array}$} & $\begin{array}{c}\text { No sterile } \\
\text { ovules }\end{array}$ & \multicolumn{5}{c}{ Type and number of embryo sacs ${ }^{1)}$} \\
\cline { 5 - 8 } T-2 & 63 & 12 & 2 & 17 & $4(3)^{2)}$ & $1(0)^{2)}$ & 27 \\
N68/96-8-0-11 & 58 & 4 & 0 & 2 & $7(4)$ & $1(0)$ & 44 \\
Natsukaze & 58 & 14 & 0 & 4 & $6(5)$ & $1(0)$ & 33 \\
T-75 & 54 & 2 & 0 & 10 & $8(6)$ & $0(0)$ & 34 \\
T-43 & 52 & 2 & 0 & 2 & $16(3)$ & $0(0)$ & 32 \\
Green panic & 40 & 2 & 0 & 0 & $6(0)$ & $0(0)$ & 34 \\
Natsuyutaka & 30 & 0 & 0 & 2 & $4(2)$ & $1(0)$ & 23 \\
\hline
\end{tabular}

"SS: only two 8- or 5-nucleate mature sexual embryo sacs in an ovule; S: only one 8-nucleate mature embryo sac in an ovule; An: one or more 4-nucleate mature apomictic embryo sac; ${ }^{2)}$ : in parentheses is the numbers of ovules containing an 8-nucleate sexual embryo sac.

apospory rate contained more embryo sacs than those with a lower rate did. Combining the results of Table 2, it could be concluded that the range of period of AESIC appearance became longer as the number of embryo sacs and apospory rate increased.

8. Types of embryo sacs in apomictic plants. Five types of embryo sacs were observed (Table 3). In facultative apomicts, most ovules showed one or more aposporous embryo sacs. Ovules containing both sexual embryo sac and aposporous ones were also observed in the seven accessions tested, which agrees with the observation by Nakajima and Mochizuki (1983). Ovules with two sexual embryo sacs were observed only in T-2. However, ovules with two sexual and one or more aposporous embryo sacs were found in four accessions. In such ovules, both sexual embryo sacs were 5-nucleate with an egg cell, two synergids and two polar nuclei, but no antipodals. Both 5- and normal 8-nucleate embryo sacs were observed in different ovules with one sexual and one or more aposporous embryo sacs in the tested accessions. T-2, T-75 and Natsukaze showed 75\% (3/4), 75\% (6/8) and $83 \%(5 / 6)$ of ovules with 8-nucleate embryo sacs higher than those of the other four accessions (Table 3 ).

\section{Discussion}

Until megasporogenesis, we observed no differences between obligate sexual and apomictic plants. After megasporegenesis, different directions are followed in embryo sac development. Sexual ovules proceeded in a manner typical of the Gramineae family, i.e., functional megaspores divided and formed mature 8-nucleate embryo sac as reported by Hanna et al. (1973). Apomictic ovules, on the other hand, showed direct formation of embryo sac, i.e. AESIC (2n) different from megaspores $(n)$ divided and formed mature 4-nucleate embryo sac.

The earliest AESIC appeared in ovule always locates in where would turn toward and finally become micropylar end (Fig. $2 \mathrm{~A}_{1}, \mathrm{~B}, \mathrm{C}$ ), as the ovary grew. The laters are distributed along the first one and being apart from it (Fig. 2D,E). To clarify the mechanism of appearance of AESIC, we selected ovary length as an index and measured the ovaries when they were observed in different AESIC appearance (Table 2). It could be concluded from the range of ovary length that AESICs do not appear together in same time. Instead, they seemed following a continuous course and appeared one by one during the period from megasporegenesis even to first embryo sac maturity. Moreover, accessions with higher frequency of apospory have plural embryo sacs (Table 1), and showed wider range of period of AESIC appearance, when the ovary length used as an index was measured (Tables 1, 2).

If we assume that the time of apomixis gene expression is just before the time of AESIC appearance, it could be considered that the more the number of embryo sacs per ovule are, the 
longer the expression period will be. Therefore, as the materials for apomixis gene cloning, the accessions containing higher numbers of embryo sacs should be advantageous for their having wider duration of AESIC appearance.

Sterile ovules with degenerated embryo sac appeared in both sexual and apomictic accessions based on the observations and quantitative analysis of ovary length (Table 2 ). It is considered from Table 2 that all four reduced megaspores are degenerated in sexual accessions, and in facultative apomictic ones neither functional megaspore development nor AESIC appearance. That the ovary length of stages of first AESIC was longer than that of functional megaspore in most accessions also indicates that apospory is initiated after megasporegenesis. And more, the ovary length staged in functional megaspore was wide and close to that staged in degeneration of embryo sac. These results also support that the development of sexual embryo sac is often terminated in many aposporous apomicts at the megaspore mother cell or megaspore stage, and the products of sexual process degenerate (Nogler 1984, Asker and Jerling 1992). It is not clear only according to cytological evidence whether the differentiation of AESIC is the direct cause of the termination of sexual megagametophyte development at this early stage. The nuclear tissue of the ovule where both sexual and apomictic processes take place, therefore, should be studied at molecular level to know this tissue and its exact role in the developmental processes of the ovule, although nothing is yet known about the similarities and differences between genes expressed during apomictic and sexual developmental pathway.

About the sexual embryo sac formation in ovules with plural embryo sacs in apomictic plants, Nakajima and Mochizuki (1983) have reported that a few ovules had two sexual embryo sacs, and that in polyembryonic ovules having both sexual and apomictic embryo sacs, number of sexual embryo sacs was limited to be one. In the present study, five types of embryo sac formation were recognized (Table 3). For the case of ovules with one or two 5(8)-nucleate embryo sacs appeared with or without 4-nucleate ones in one ovule, two pathwayes could be considered that 1) the sexual embryo sac formation results from the direct division of one or two megaspore(s) though the AESIC(s) appeared (or not) in the same ovules; and that 2) it is derived from AESIC(s). Especially, as the ovules with two megaspores in chalazal end were not observed in this study while AESIC(s) appeared in the micropylar end, the former pathway could be hardly considered as a putative one. To explain the appearance of ovules containing one or two sexual embryo sacs with or without 4-nucleate apomictic ones, the later, however, seems reasonable based on that AESICs develop into not only 4-nucleate but also, at a low frequency, 5-nucleate embryo sacs in Panicum (Nakajima and Mochizuki 1983), or 8-nucleate ones in Hieracium (Nogler 1984). In normal sexual embryo sac formation, two polar nuclei derived from one of four micropylar nuclei and one of four chalazal nuclei, were distinguished in this study. For the 5-nucleate embryo sac formation, it could be imaged that after megaspore or AESIC divided into two nuclei, only the micropylar nucleus continued to divide twice and to form 4-nuclei, the chalazal nucleus and one of the four micropylar nuclei pair with each other to form two polar nuclei. On the other hand, a rare case of 4-nucleate embryo sac derived from AESIC, with an egg cell, one synergid cell and two polar nuclei was also depicted for Panicum by Bashaw and Hanna (1990). Combining the above reports and the observation of embryo sac formation in this study, it could be concluded that facultatively apomictic guineagrass produces not only common and rare Panicum-type (4-nucleate), but also its typical type (5-nucleate), and in addition, Polygonum-type (8-nucleate), in one ovule.

\section{Summary}

Seven facultatively apomictic accessions and three obligately sexual accessions of guineagrass (Panicum maximum) were studied cytologically and quantitatively for clarifying the 
mechanisms of appearance of aposporous embryo sac initial cell (AESIC) and apomictic embryo sac formation by using ovary length as an index. The observations of AESIC appearance and their development indicates that most of ovules in apomictic accessions contain several AESICs, and the number of AESICs increased as the ovary grew before anthesis. That is, several AESICs in the same ovule did not differentiate synchronously, but instead, they seemed following a continuous course and appeared one by one during the period from after megasporegenesis to the first AESIC-derived embryo sac maturity. It was also found that the higher the frequency of apospory was, the greater the number of AESICs was, and the longer the duration of AESIC appearance should be. This result will produce the information on sampling of the materials used for the apomixis gene isolation program. The type of embryo sacs in same ovules of apomictic plants were also distinguished and discussed.

\section{Acknowledgement}

The authors thank Dr. A. Kojima and Mr. S. Kato (Laboratory of Plant Genetic Resources, Kyushu National Agricultural Experiment Station) for giving useful advice and support to this study. Grateful also to Prof. Dr. F. Hoffmann (University of California, Irine) for his critical reading of this manuscript.

\section{References}

Asker, S. E. and Jerling, L. 1992. Apomixis in Plants. CRC Press, Boca Raton.

Bashaw, E. C. and Hanna, W. W. 1990. Apomictic reproduction. In Reproductive Versatility in the Grasses, G. P. Chapman, ed. (Cambridge: Cambridge University Press), pp. 100-130.

Dujardin, M. and Hanna, W. W. 1983. Apomictic and sexual pearl millet $\times$ Pennisetum squamulatum hybrids. J. Hered. 74: 277-279.

- and - 1985. Cytology and reproduction of reciprocal backcrosses between pearl millet and sexual and apomictic hybrids of pearl millet $\times$ Pennisetum squamulatum. Crop Sci. 25: 55-62.

Hann, W. W. and Bashaw, E. C. 1987. Apomixis: Its identification and use in plant breeding. Crop Sci. 27: 1136-1139.

-, Powell, J., Millot, J. C. and Burton, G. W. 1973. Cytology of obligate sexual plants in Panicum maximum Jacq. and their use in controlled hybrids. Crop Sci. 13: 695-697.

Herr, J. M. Jr. 1982. An analysis of methods for permanently mounting ovules cleared in four-and-a-half type clearing fluids. Stain. Technol. 57: 161-169.

Kojima, A. and Nagato, Y. 1992. Diplosporous embryo-sac formation and the degree of diplospory in Allium tuberosum. Sex. Plant Reprod. 5: 72-78.

Nakagawa, H. 1990. Embryo sac analysis and crossing procedure for breeding apomictic guineagrass (Panicum maximum Jacq.). JARQ 24: 163-168.

Nakajima, K. and Mochizuki, N. 1983. Degrees of sexual plants of guineagrass by the simplified embryo sac analysis. Japan. J. Breed. 33: 45-54.

Nogler, G. A. 1984. Gametophytic apomixis. In Embryology of Angiosperms, B. M. Johri, ed. (Berlin: SpringerVerlag), pp. 475-518.

Ozias-Akins, P., Lubbers, E. L., Hanna, W. W. and McNay, J. W. 1993. Transmission of the apomictic mode of reproduction in Pennisetum: co-inheritance of the trait and molecular markers. Theor. Appl. Genet. 85: 632638.

Savidan, Y. 1975. Hérédité de l'apomixie, contribution a l'étude de l'héredite de l'apomixie sur Panicum maximum Jacq. (analyse des sacs embryonnaires). Cah. ORSTOM, sér. Biol. 10: 91-95.

- and Pernès, J. 1982. Diploid-tetraploid-dihaploid cycles and the evolution of Panicum maximum Jacq. Evolution 36: $596-600$.

Smith, R. L. 1972. Sexual reproduction in Panicum maximum Jacq. Crop Sci. 12: 624-627.

Warmke, H. E. 1954. Apomixis in Panicum maximum. Amer. J. Bot. 41: 5-11. 